\title{
Factorizations of some lower triangular matrices and related combinatorial identities
}

\author{
Cahit Köme \\ Department of Information Technology, \\ Nevşehir Hacı Bektaş Veli University \\ 50300, Turkey \\ e-mail: cahitenevsehir.edu.tr
}

Received: 29 January 2021

\begin{abstract}
In this study, we investigate the connection between second order recurrence matrix and several combinatorial matrices such as generalized $r$-eliminated Pascal matrix, Stirling matrix of the first and of the second kind matrices. We give factorizations and inverse factorizations of these matrices by virtue of the second order recurrence matrix. Moreover, we derive several combinatorial identities which are more general results of some earlier works.
\end{abstract}

Keywords: Pascal matrix, Stirling matrix, Second order recurrence matrix, Factorization, Combinatorial identities.

2010 Mathematics Subject Classification: 15A23, 11B39, 05A10.

\section{Introduction}

Infinite lower triangular matrices arise in computer science, numerical analysis, combinatorics, physics, several branches of science and different mathematical disciplines. In recent years, lower triangular matrices have attracted the attention of some researchers [1-3, 5, 7-15]. For example, Brawer and Pirovino investigated the Cholesky factorization of the Pascal matrix [3]. They produced some combinatorial identities and an existence theorem for Diophantine equation system. Cheon and Kim obtained the Pascal-type matrices from Stirling numbers of the first kind $s(n, k)$ and of the second kind $S(n, k)$ and they derived some combinatorial identities via matrix representation of the Stirling numbers and generalized these matrices in one or two variables [5]. Lee et al. obtained the Pascal matrix and the Stirling matrices of the first kind and the second kind 
from the Fibonacci matrix. They also presented some combinatorial identities from the matrix representation of the Pascal matrix, the Stirling matrices of the first kind and the second kind and the Fibonacci matrix [9]. Zhang and Wang gave another factorization of the Pascal matrix, which was apparently missed by Lee et al [9], via Fibonacci matrix and presented some identities using these matrices [14]. Zhang et al. studied the Lucas matrix and they derived some identities involving the Lucas numbers the matrix representation of generalized Pascal matrix and the Lucas matrix [15]. Quintana et al. introduced the generalized Apostol-type polynomial matrix and they provided some factorizations of the Apostol-type polynomial matrix involving the generalized Pascal matrix, Fibonacci and Lucas matrices [10]. Irmak and Köme investigated the Cholesky factorization of the symmetric Lucas matrix and obtained the upper and lower bounds for the eigenvalues of the symmetric Lucas matrix by using some majorization techniques [7]. Bayat and Teimoori studied the $r$-eliminated Pascal matrix and they investigated the relationship between these matrix and Cesàro matrix [2].

For any natural numbers $n$ and $r$ and real numbers $x$ and $y$, the $n \times n$ lower triangular generalized $r$-eliminated functional Pascal matrix $\mathscr{P}_{n}[r ; x, y]=\left[p_{i j}\right]$ and its inverse $\mathscr{P}_{n}^{-1}[r ; x, y]=\left[p_{i j}^{\prime}\right]$ are defined by

$$
p_{i j}=\left\{\begin{array}{ll}
\left(\begin{array}{c}
i+r-1 \\
j+r-1
\end{array}\right) x^{i-j} y^{i+j-2}, & \text { if } i \geqslant j, \\
0 & \text { otherwise }
\end{array} \text { and } p_{i j}^{\prime}=\left\{\begin{array}{ll}
(-1)^{i-j}\left(\begin{array}{c}
i+r-1 \\
j+r-1
\end{array}\right) x^{i-j} y^{2-i-j}, & \text { if } i \geqslant j, \\
0, & \text { otherwise }
\end{array},\right.\right.
$$

where the binomial coefficient $\left(\begin{array}{l}i \\ j\end{array}\right)$ counts the number of lattice paths from $(0,0)$ to $(i-j, j)$ with steps $(1,0)$ and $(0,1)$.

For example, if we take $n=4$, we get

$$
\mathscr{P}_{4}[r ; x, y]=\left[\begin{array}{cccc}
1 & 0 & 0 & 0 \\
(r+1) x y & y^{2} & 0 & 0 \\
\frac{1}{2}(r+1)(r+2) x^{2} y^{2} & (r+2) x y^{3} & y^{4} & 0 \\
\frac{1}{6}(r+1)(r+2)(r+3) x^{3} y^{3} & \frac{1}{2}(r+2)(r+3) x^{2} y^{4} & (r+3) x y^{5} & y^{6}
\end{array}\right]
$$

and

$$
\mathscr{P}_{4}^{-1}[r ; x, y]=\left[\begin{array}{cccc}
1 & 0 & 0 & 0 \\
-\frac{(r+1) x}{y} & \frac{1}{y^{2}} & 0 & 0 \\
\frac{(r+1)(r+2) x^{2}}{2 y^{2}} & -\frac{(r+2) x}{y^{3}} & \frac{1}{y^{4}} & 0 \\
-\frac{(r+1)(r+2)(r+3) x^{3}}{6 y^{3}} & \frac{(r+2)(r+3) x^{2}}{2 y^{4}} & -\frac{(r+3) x}{y^{5}} & \frac{1}{y^{6}}
\end{array}\right] \text {. }
$$

For nonnegative integers $n, k$ and $n \geqslant k \geqslant 0$, the Stirling numbers of the first kind $s(n, k)$ and of the second kind $S(n, k)$, which describe the coefficients of polynomials that commonly arise in combinatorial problems, are defined as the coefficients in the following expansion of a variable $x$ (see [4]):

$$
[x]_{n}=\sum_{k=0}^{n}(-1)^{n-k} s(n, k) x^{k} \text { and } x^{n}=\sum_{k=0}^{n} S(n, k)[x]_{k},
$$


where

$$
[x]_{n}=\left\{\begin{array}{ll}
x(x-1) \cdots(x-n+1), & \text { if } n \geqslant 1, \\
1, & \text { if } n=0
\end{array} .\right.
$$

Moreover, the $S(n, k)$ satisfy the following formula

$$
S(n, k)=\sum_{l=k-1}^{n-1}\left(\begin{array}{c}
n-1 \\
l
\end{array}\right) S(l, k-1) .
$$

The Stirling numbers of the second kind and of the first kind can be expressed with binomial sums as

$$
S(m, n)=\sum_{k=0}^{n} \frac{\left(\begin{array}{l}
n \\
k
\end{array}\right) k^{m}}{n !(-1)^{k-n}}
$$

and

$$
s(n, m)=\sum_{k=0}^{n-m}\left[(-1)^{k}\left(\begin{array}{c}
n-1+k \\
n-m+k
\end{array}\right)\left(\begin{array}{c}
2 n-m \\
n-m-k
\end{array}\right) S(n-m+k, k)\right],
$$

respectively. For the Stirling numbers $s(i, j)$ and $S(i, j)$ of the first kind and of the second kind, respectively, define $\mathscr{S}_{n}^{(1)}=\left[s_{i j}\right]$ and $\mathscr{S}_{n}^{(2)}=\left[S_{i j}\right]$ to be the $n \times n$ matrices by

$$
s_{i j}=\left\{\begin{array}{ll}
s(i, j) & \text { if } i \geqslant j, \\
0 & \text { otherwise }
\end{array} \quad \text { and } \quad S_{i j}=\left\{\begin{array}{ll}
S(i, j) & \text { if } i \geqslant j \\
0 & \text { otherwise }
\end{array} .\right.\right.
$$

We call the matrices $\mathscr{S}_{n}^{(1)}$ and $\mathscr{S}_{n}^{(2)}$ Stirling matrix of the first kind and of the second kind, respectively (see [6]). For example,

$$
\mathscr{S}_{5}^{(1)}=\left[\begin{array}{ccccc}
1 & 0 & 0 & 0 & 0 \\
-1 & 1 & 0 & 0 & 0 \\
2 & -3 & 1 & 0 & 0 \\
-6 & 11 & -6 & 1 & 0 \\
24 & -50 & 35 & -10 & 1
\end{array}\right] \text { and } \mathscr{S}_{5}^{(2)}=\left[\begin{array}{ccccc}
1 & 0 & 0 & 0 & 0 \\
1 & 1 & 0 & 0 & 0 \\
1 & 3 & 1 & 0 & 0 \\
1 & 7 & 6 & 1 & 0 \\
1 & 15 & 25 & 10 & 1
\end{array}\right] \text {. }
$$

In recents years, some researchers studied the second order binary recurrences with lower triangular matrices $[11,12]$. Stănică extended some results on the factorization of matrices associated to Lucas, Pascal, Stirling sequences by the Fibonacci matrix and then he gave the Cholesky factorization for the symmetric matrix associated to $r$-order recurrent sequences [11]. Stanimirović et al. defined the matrix $U_{n}^{(a, b, s)}$ whose elements consist of the non-degenerated second order recurrent sequence. The authors also considered various correlations between the matrix $U_{n}^{(a, b, s)}$ and the Pascal matrix of the first and the second kind [12].

The non-degenerated second order binary recurrence sequence is defined by

$$
W_{n}=p W_{n-1}+q W_{n-2}, W_{0}=a, W_{1}=b,
$$

where $a, b, p, q$ are nonzero positive real numbers and $\Delta=\sqrt{p^{2}+4 q} \neq 0$. The Binet formula of the second order binary recurrences is $W_{n}=A \alpha^{n}-B \beta^{n}$ where $\alpha=\frac{1}{2}\left(p+\sqrt{p^{2}+4 q}\right)$, $\beta=\frac{1}{2}\left(p-\sqrt{p^{2}+4 q}\right)$ and $A=\frac{b-a \beta}{\alpha-\beta}, B=\frac{b-a \alpha}{\alpha-\beta}$. 
The $n \times n$ lower triangular second order binary recurrence matrix $\mathscr{W}_{n}=\left[w_{i j}\right]$ and its inverse $\mathscr{W}_{n}^{-1}=\left[w_{i j}^{\prime}\right]$ are defined, in [12], as

$$
w_{i j}=\left\{\begin{array}{ll}
W_{i+j-1}, & i \geqslant j, \\
0, & i<j
\end{array} \text { and } w_{i j}^{\prime}= \begin{cases}\frac{(-1)^{i-j} a^{2} q+a b p-b^{2}}{b^{i-j+1}} a^{i-j-2} q^{i-j-1}, & i \geqslant j+2 \\
-\frac{a q+b p}{b^{2}}, & i=j+1 \\
\frac{1}{b}, & i=j \\
0, & i<j\end{cases}\right.
$$

The relations between the Pascal matrix, Stirling matrices and Fibonacci matrix motivate us to study a more generalized situation. Thus, inspiring by the above cited works, we investigate the relations between the matrix $\mathscr{W}_{n}$ and $\mathscr{P}_{n}[r ; x, y], \mathscr{S}_{n}^{(1)}$ and $\mathscr{S}_{n}^{(2)}$. By eliminating the $r$-th column from $\mathscr{P}_{n}[r ; x, y]$, we present two factorizations of $\mathscr{P}_{n}[r ; x, y]$ via $\mathscr{W}_{n}$. Moreover, we give the factorizations of the matrices $\mathscr{S}_{n}^{(1)}$ and $\mathscr{S}_{n}^{(2)}$ by means of $\mathscr{W}_{n}$. We also derive several combinatorial identities using these relations and provide more general results.

\section{First factorization of the generalized $r$-eliminated Pascal matrix}

In this section, we consider the first factorization of the generalized $r$-eliminated Pascal matrix by means of the second order recurrence matrix.

Definition 1. For any nonzero positive real numbers $a, b, c, d$ and any natural number $n$, an $n \times n$ matrix $\Phi_{n}[r ; x, y]=\left[\varphi_{i j}\right]$ is defined by

$$
\begin{aligned}
\varphi_{i j}=x^{-j} y^{j-2} & {\left[\frac{(x y)^{i}}{b}\left(\begin{array}{l}
i+r-1 \\
j+r-1
\end{array}\right)-(x y)^{i-1} \frac{a q+b p}{b^{2}}\left(\begin{array}{l}
i+r-2 \\
j+r-1
\end{array}\right)\right.} \\
& \left.+\sum_{k=j}^{i-2}\left[(-1)^{i-k} \frac{a^{i-k-2} q^{i-k-1}(x y)^{k}}{b^{i-k+1}}\left(a^{2} q+a b p-b^{2}\right)\left(\begin{array}{c}
k+r-1 \\
j+r-1
\end{array}\right)\right]\right] .
\end{aligned}
$$

Theorem 2.1. Let $\Phi_{n}[r ; x, y]$ is the matrix as in (9). For the generalized r-eliminated Pascal matrix $\mathscr{P}_{n}[r ; x, y]$ and the second order recurrence matrix $\mathscr{W}_{n}$, we have

$$
\mathscr{P}_{n}[r ; x, y]=\mathscr{W}_{n} \Phi_{n}[r ; x, y]
$$

where $x \neq 0, y \neq 0$ and $b \neq 0$.

Proof. In order to prove the theorem, it is sufficient to verify that $\mathscr{W}_{n}^{-1}[p, q ; a, b] \mathscr{P}_{n}[r ; x, y]=$ $\Phi_{n}[r ; x, y]$. By virtue of the Definition 1 , we see that $\varphi_{i j}=0$ for $i<j$. So, we will consider the cases $i \geqslant j$. For $i=j$, we have $\varphi_{j j}=w_{j j}^{\prime} p_{j j}=\frac{y^{2(j-1)}}{b}$. For $i=j+1$, we get $\varphi_{j+1, j}=$ $w_{j+1, j}^{\prime} p_{j j}+w_{j+1, j+1}^{\prime} p_{j+1, j}=\frac{y^{2(j-1)}(b(r+j) x y-a q-b p)}{b^{2}}$. For $i \geqslant j+2$, we have $\varphi_{i j}=w_{i i}^{\prime} p_{i j}+$ $w_{i, i-1}^{\prime} p_{i-1, j}+\sum_{k=j}^{i-2} w_{i k}^{\prime} p_{k j}$, which is of the form (9). Hence the proof is completed. 
Here we note that the following results are the special cases of Theorem 2.1:

- if we take $r=0$ and $y=1$ in Definition 2.1, we obtain Theorem 3.1 in [12],

- if we take $a=0, b=1, x=1, y=1, p=1, q=1$ and $r=0$ in Definition 2.1, we obtain Equation (3) in [9],

- if we take $a=2, b=1, y=1, p=1, q=1$ and $r=0$ in Definition 2.1, we obtain Theorem 3.1 in [15].

Furthermore, by virtue of Theorem 2.1, we can derive the following corollary.

Corollary 2.1.1. For $i \geqslant j+2$, we have

$$
\begin{aligned}
\left(\begin{array}{c}
i+r-1 \\
j+r-1
\end{array}\right) x^{i-j} y^{i+j-2}= & W_{i-j+1} \frac{y^{2(j-1)}}{b}+W_{i-j} \frac{y^{2(j-1)}(b(r+j) x y-a q-b p)}{b^{2}} \\
& +\sum_{m=j+2}^{i} W_{i-m+1} x^{-j} y^{j-2}\left[\frac{(x y)^{m}}{b}\left(\begin{array}{c}
m+r-1 \\
j+r-1
\end{array}\right)-(x y)^{m-1} \frac{a q+b p}{b^{2}}\left(\begin{array}{c}
m+r-2 \\
j+r-1
\end{array}\right)\right. \\
& \left.+\sum_{k=j}^{m-2}(-1)^{m-k} \frac{(x y)^{k}\left(a^{2} q+a b p-b^{2}\right)\left(\frac{a q}{b}\right)^{m-k-1}}{a b^{2}}\left(\begin{array}{c}
k+r-1 \\
j+r-1
\end{array}\right)\right],
\end{aligned}
$$

where $x \neq 0, y \neq 0$ and $b \neq 0$.

Proof. Since $\mathscr{P}_{n}[r ; x, y]=\mathscr{W}_{n} \Phi_{n}[r ; x, y]$, we observe that $p_{i j}=0$ for $i<j$. So we will consider the another cases. For $i=j$ and $i=j+1$, we have

$$
p_{j j}=W_{1} \varphi_{j j}=b \frac{y^{2(j-1)}}{b}=y^{2(j-1)}
$$

and

$$
\begin{aligned}
p_{j+1, j} & =W_{2} \varphi_{j j}+W_{1} \varphi_{j+1, j} \\
& =\frac{y^{2 j-2}(a q+b p)}{b}+\frac{y^{2 j-2}(-a q+b x y(j+r)-b p)}{b} \\
& =x y^{2 j-1}(j+r) .
\end{aligned}
$$

By virtue of (9), in the last case, $i \geqslant j+2$, we get

$$
p_{i j}=W_{i-j+1} \varphi_{j j}+W_{i-j} \varphi_{j+1, j}+\sum_{m=j+2}^{i} W_{i-m+1} \varphi_{m j}
$$

which verifies Corollary 2.1.1.

From the definition of (9), we have the inverse matrix $\Phi_{n}^{-1}=\left[\varphi_{i j}^{\prime}\right]$, of $\Phi_{n}$, where

$$
\varphi_{i j}^{\prime}=\sum_{k=j}^{i}(-1)^{i+k}\left(\begin{array}{c}
i+r-1 \\
k+r-1
\end{array}\right) x^{i-k} y^{2-i-k} W_{k-j+1} .
$$

Since $\mathscr{W}_{n}=\mathscr{P}_{n}[r ; x, y] \Phi_{n}[r ; x, y]^{-1}$, we have

$$
w_{n k}=p_{n 1} \varphi_{1 k}^{\prime}+p_{n 2} \varphi_{2 k}^{\prime}+\ldots+p_{n, n-1} \varphi_{n-1, k}^{\prime}+p_{n n} \varphi_{n k}^{\prime} .
$$

Hence, by virtue of (1) and (11), we get

$$
W_{n-k+1}=\sum_{t=k}^{n}\left[\left(\begin{array}{c}
n+r-1 \\
t+r-1
\end{array}\right) x^{n-t} y^{n+t-2}\left[\sum_{s=k}^{t}(-1)^{t+s}\left(\begin{array}{c}
t+r-1 \\
s+r-1
\end{array}\right) x^{t-s} y^{2-t-s} W_{s-k+1}\right]\right] \text {. }
$$


Now, we consider factorization of $\mathscr{W}_{n}$. We define an $n \times n$ matrix $X_{n}=\left[x_{i j}\right]$ as

$$
x_{i j}=\left\{\begin{array}{ll}
W_{i}, & j=1, \\
1, & i=j, \\
0, & \text { otherwise }
\end{array} \quad \text { i.e., } X_{n}=\left[\begin{array}{cccc}
W_{1} & 0 & \cdots & 0 \\
W_{2} & 1 & \cdots & 0 \\
\vdots & \vdots & \ddots & \vdots \\
W_{n} & 0 & \cdots & 1
\end{array}\right]\right.
$$

The next theorem follows by a simple calculation.

Theorem 2.2. For $n \geqslant 1$, we have

$$
\mathscr{W}_{n}=X_{n}\left(I_{1} \oplus X_{n-1}\right)\left(I_{2} \oplus X_{n-2}\right) \cdots\left(I_{n-1} \oplus X_{1}\right) .
$$

For example, for $n=5$, we get

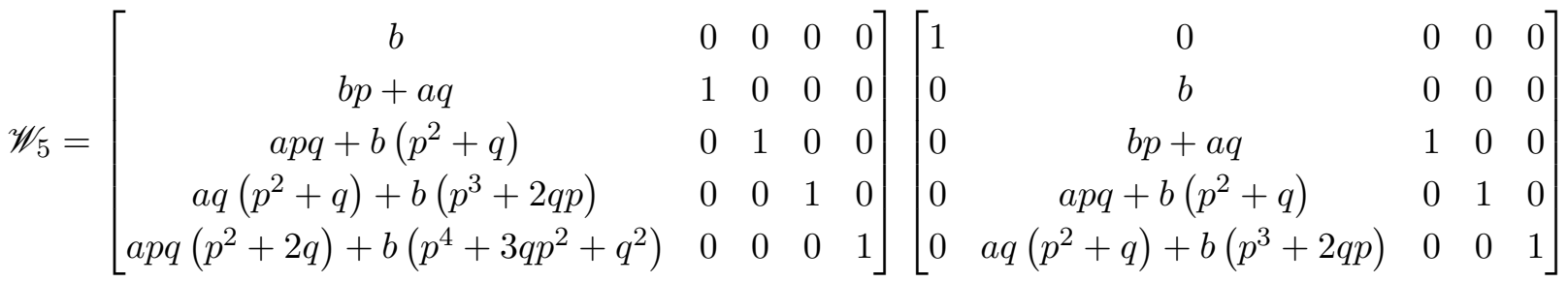

$$
\begin{aligned}
& {\left[\begin{array}{ccccc}
1 & 0 & 0 & 0 & 0 \\
0 & 1 & 0 & 0 & 0 \\
0 & 0 & b & 0 & 0 \\
0 & 0 & b p+a q & 1 & 0 \\
0 & 0 & a p q+b\left(p^{2}+q\right) & 0 & 1
\end{array}\right]\left[\begin{array}{ccccc}
1 & 0 & 0 & 0 & 0 \\
0 & 1 & 0 & 0 & 0 \\
0 & 0 & 1 & 0 & 0 \\
0 & 0 & 0 & b & 0 \\
0 & 0 & 0 & b p+a q & 1
\end{array}\right]\left[\begin{array}{ccccc}
1 & 0 & 0 & 0 & 0 \\
0 & 1 & 0 & 0 & 0 \\
0 & 0 & 1 & 0 & 0 \\
0 & 0 & 0 & 1 & 0 \\
0 & 0 & 0 & 0 & b
\end{array}\right]} \\
& =\left[\begin{array}{cccrr}
b & 0 & 0 & 0 & 0 \\
b p+a q & b & 0 & 0 & 0 \\
a p q+b\left(p^{2}+q\right) & b p+a q & b & 0 & 0 \\
a q\left(p^{2}+q\right)+b\left(p^{3}+2 q p\right) & a p q+b\left(p^{2}+q\right) & b p+a q & b & 0 \\
a p q\left(p^{2}+2 q\right)+b\left(p^{4}+3 q p^{2}+q^{2}\right) & a q\left(p^{2}+q\right)+b\left(p^{3}+2 q p\right) & a p q+b\left(p^{2}+q\right) & b p+a q & b
\end{array}\right]
\end{aligned}
$$

Before giving factorization of the shifted Pascal matrix we need to factorize $\Phi_{n}$. So, we define $n \times n$ matrices $H_{n}$ and $\bar{H}_{i}$ as

$$
H_{n}=\left[\begin{array}{ccccc}
\frac{1}{b} & 0 & 0 & \cdots & 0 \\
\frac{b(r+1) x y-a q-b p}{b^{2}} & y^{2} & 0 & \cdots & 0 \\
\vdots & \vdots & \ddots & \ddots & \vdots \\
\varphi_{n-1,1} & x^{n-3} y^{n-1} & \cdots & y^{2} & 0 \\
\varphi_{n 1} & x^{n-2} y^{n} & \cdots & x y^{3} & y^{2}
\end{array}\right] \text { and } \bar{H}_{i}=I_{n-i} \oplus H_{i}
$$

where $\varphi_{i j}$ is defined in (9). Hence, we can give the factorization of $\Phi_{n}$ with the following theorem.

Theorem 2.3. The matrix $\Phi_{n}$ can be factored by the $\bar{H}_{i}$ 's as follows:

$$
\Phi_{n}=\bar{H}_{n} \bar{H}_{n-1} \bar{H}_{n-2} \ldots \bar{H}_{2} \bar{H}_{1} \text {. }
$$

Now, we can give the factorization of $r$-eliminated Pascal matrix with the following theorem by virtue of Theorem 2.2 and 2.3.

Theorem 2.4. For the $n \times n$ generalized $r$-eliminated Pascal matrix, we have

$$
\mathscr{P}_{n}[r ; x, y]=\prod_{i=0}^{n-1}\left(I_{i} \oplus X_{n-i}\right) \prod_{i=1}^{n} \bar{H}_{n-i+1} .
$$




\section{Second factorization of the $r$-eliminated functional Pascal matrix}

In this section, we consider the second factorization of the $r$-eliminated functional Pascal matrix by means of the second order recurrence matrix.

Definition 2. For any nonzero positive real numbers $a, b, c, d$ and any natural number $n$, an $n \times n$ matrix $\Psi_{n}[r ; x, y]=\left[\psi_{i j}\right]$ is defined by

$$
\begin{aligned}
\psi_{i j}=( & x y)^{i}\left[\frac{x^{-j} y^{j-2}}{b}\left(\begin{array}{c}
i+r-1 \\
j+r-1
\end{array}\right)-\frac{x^{-j-1} y^{j-1}(a q+b p)}{b^{2}}\left(\begin{array}{c}
i+r-1 \\
j+r
\end{array}\right)\right. \\
& \left.+\sum_{k=j+2}^{i}\left[\frac{(-1)^{k-j} x^{-k} y^{k-2} a^{-j+k-2} q^{-j+k-1}}{b^{-j+k+1}}\left(a^{2} q+a b p-b^{2}\right)\left(\begin{array}{c}
i+r-1 \\
k+r-1
\end{array}\right)\right]\right] .
\end{aligned}
$$

Theorem 3.1. Let $\Psi_{n}[r ; x, y]$ is the matrix as in (17). For the r-eliminated functional Pascal matrix $\mathscr{P}_{n}[r ; x, y]$ and the second order recurrence matrix $\mathscr{W}_{n}$, we have

$$
\mathscr{P}_{n}[r ; x, y]=\Psi_{n}[r ; x, y] \mathscr{W}_{n}
$$

where $x \neq 0, y \neq 0$ and $b \neq 0$.

Proof. In order to prove the theorem, it is sufficient to verify that $\mathscr{P}_{n}[r ; x, y] \mathscr{W}_{n}^{-1}=\Psi_{n}[r ; x, y]$. From Definition 2, we observe that $\psi_{i j}=0$ for $i<j$. So, we will consider the cases $i \geqslant j$.

- For $i=j$, we have $\psi_{j j}=p_{j j} w_{j j}^{\prime}=\frac{y^{2(j-1)}}{b}$.

- For $i=j+1$, we get

$$
\psi_{j+1, j}=p_{j+1, j} w_{j j}^{\prime}+p_{j+1, j+1} w_{j+1, j}^{\prime}=\frac{y^{2 j-1}(b x(j+r)-y(a q+b p))}{b^{2}} .
$$

- For $i \geqslant j+2$, we have $\psi_{i j}=p_{i i} w_{i j}^{\prime}+p_{i, i-1} w_{i-1, j}^{\prime}+\sum_{k=j}^{i-2} p_{i k} w_{k j}^{\prime}$ which satisfies (17).

Hence the proof is completed.

By virtue of Theorem 3.1, we have the following corollary.

Corollary 3.1.1. For $i \geqslant j+2$, we have

$$
\begin{aligned}
& \left(\begin{array}{c}
i+r-1 \\
j+r-1
\end{array}\right) x^{i-j} y^{i+j-2} \\
& =\frac{y^{2(i-1)}}{b} W_{i-j+1}+\frac{y^{2 i-3}(b x(i+r-1)-y(a q+b p))}{b^{2}} W_{i-j} \\
& \quad+\sum_{m=j}^{i-2}(x y)^{i}\left[\frac{x^{-m} y^{m-2}}{b}\left(\begin{array}{c}
i+r-1 \\
m+r-1
\end{array}\right)-\frac{x^{-m-1} y^{m-1}(a q+b p)}{b^{2}}\left(\begin{array}{c}
i+r-1 \\
m+r
\end{array}\right)\right. \\
& \left.\quad+\sum_{k=m+2}^{i} \frac{(-1)^{k-m} x^{-k} y^{k-2} a^{k-m-2} q^{k-m-1}}{b^{k-m+1}}\left(a^{2} q+a b p-b^{2}\right)\left(\begin{array}{c}
i+r-1 \\
k+r-1
\end{array}\right)\right] W_{m-j+1},
\end{aligned}
$$

where $x \neq 0, y \neq 0$ and $b \neq 0$. 
Proof. Since $\mathscr{P}_{n}[r ; x, y]=\Psi_{n}[r ; x, y] \mathscr{W}_{n}$, we observe that $p_{i j}=0$ for $i<j$. So we will consider the another cases. For $i=j$ and $i=j+1$, we have

$$
p_{j j}=\psi_{j j} W_{1}=y^{2(j-1)}
$$

and

$$
\begin{aligned}
p_{j+1, j} & =\psi_{j+1, j+1} W_{2}+\psi_{j+1, j} W_{1} \\
& =\frac{y^{2 j-2}(a q+b p)}{b}+\frac{y^{2 j-1}(b x(j+r)-y(a q+b p))}{b} \\
& =x y^{2 j-1}(j+r) .
\end{aligned}
$$

For the last case $i \geqslant j+2$, from (17), we get

$$
p_{i j}=\psi_{i i} W_{i-j+1}+\psi_{i, i-1} W_{i-j}+\sum_{m=j}^{i-2} \psi_{i, m} W_{m-j+1}
$$

which verifies (19). Thus, the proof is completed.

From the definition of (17), we have the inverse matrix $\Psi_{n}^{-1}=\left[\psi_{i j}^{\prime}\right]$, of $\Psi_{n}$, where

$$
\psi_{i j}^{\prime}=\sum_{k=j}^{i} W_{i-k+1}(-1)^{k+j}\left(\begin{array}{c}
k+r-1 \\
j+r-1
\end{array}\right) x^{k-j} y^{2-k-j} .
$$

Since $\mathscr{W}_{n}=\Psi_{n}[r ; x, y]^{-1} \mathscr{P}_{n}[r ; x, y]$, we have

$$
w_{n k}=\psi_{n 1}^{\prime} p_{1 k}+\psi_{n 2}^{\prime} p_{2 k}+\ldots+\psi_{n, n-1}^{\prime} p_{n-1, k}+\psi_{n n}^{\prime} p_{n k} .
$$

Hence, by virtue of (1) and (20), we get

$$
W_{n-k+1}=\sum_{t=k}^{n}\left[\sum_{s=t}^{n} W_{n-s+1}(-1)^{s+t}\left(\begin{array}{c}
s+r-1 \\
t+r-1
\end{array}\right) x^{s-t} y^{2-s-t}\right]\left(\begin{array}{c}
t+r-1 \\
k+r-1
\end{array}\right) x^{t-k} y^{t+k-2} .
$$

Now, we define $n \times n$ matrices $L_{n}$ and $\bar{L}_{i}$ as

$$
L_{n}=\left[\begin{array}{ccccc}
\frac{1}{b} & 0 & 0 & \cdots & 0 \\
\frac{y(b(r+1) x-y(a q+b p))}{b^{2}} & y^{2} & 0 & \cdots & 0 \\
\vdots & \vdots & \ddots & \ddots & \vdots \\
\psi_{n-1,1} & x^{n-3} y^{n-1} & \cdots & y^{2} & 0 \\
\psi_{n 1} & x^{n-2} y^{n} & \cdots & x y^{3} & y^{2}
\end{array}\right], \text { and } \bar{L}_{i}=I_{n-i} \oplus L_{i}
$$

where $\psi_{i j}$ is defined in (17). Thus, we can give the factorization of $\Psi_{n}$ with the following theorem.

Theorem 3.2. The matrix $\Psi_{n}$ can be factored by the $\bar{L}_{i}$ 's as follows:

$$
\Psi_{n}=\bar{L}_{n} \bar{L}_{n-1} \bar{L}_{n-2} \cdots \bar{L}_{2} \bar{L}_{1} .
$$

Now, we can give the factorization of $r$-eliminated Pascal matrix with the following theorem by virtue of Theorem 2.2 and 3.2.

Theorem 3.3. For the $n \times n$ generalized $r$-eliminated Pascal matrix, we have

$$
\mathscr{P}_{n}[r ; x, y]=\prod_{i=1}^{n} \bar{L}_{n-i+1} \prod_{i=0}^{n-1}\left(I_{i} \oplus X_{n-i}\right)
$$




\section{Stirling matrices via second order binary recurrence matrix}

In this section, we consider relationships between Stirling matrix of the second kind and the second order binary recurrences.

Definition 3. For any nonzero positive real numbers $a, b, c, d$ and any natural number $n$, an $n \times n$ matrix $\mathscr{M}_{n}=\left[\mu_{i j}\right]$ is defined by

$$
\mu_{i j}=\frac{1}{b} S(i, j)-\frac{(a q+b p)}{b^{2}} S(i-1, j)+\sum_{k=j}^{i-2} \frac{(-1)^{i-k} a^{i-k-2} q^{i-k-1}\left(a^{2} q+a b p-b^{2}\right)}{b^{i-k+1}} S(k, j) .
$$

Theorem 4.1. Let $\mathscr{M}_{n}$ is the matrix as in (24). For the Stirling matrix of the second kind and second order recurrence matrix $\mathscr{W}_{n}$, we have

$$
\mathscr{S}_{n}^{(2)}=\mathscr{W}_{n} \mathscr{M}_{n}
$$

Proof. In order to prove the theorem, it is sufficient to verify $\mathscr{W}_{n}^{-1} \mathscr{S}_{n}^{(2)}=\mathscr{M}_{n}$. By virtue of the Definition 3, we observe that $\mu_{i j}=0$ for $i<j$. So, we will consider the cases $i \geqslant j$. The cases $i=j$ and $i=j+1$ can be verified as:

$$
\mu_{j j}=w_{j j}^{\prime} S(j, j)=\frac{1}{b}
$$

and

$$
\begin{aligned}
\mu_{j+1, j} & =w_{j+1, j}^{\prime} S(j, j)+w_{j+1, j+1}^{\prime} S(j+1, j) \\
& =\frac{1}{b} \sum_{k=0}^{j} \frac{\left(\begin{array}{l}
j \\
k
\end{array}\right) k^{j+1}}{j !(-1)^{k-j}}-\frac{(a q+b p)}{b^{2}} .
\end{aligned}
$$

For the last case, $i \geqslant j+2$, we get

$$
\begin{aligned}
\mu_{i j} & =w_{i i}^{\prime} S(i, j)+w_{i, i-1}^{\prime} S(i-1, j)+\sum_{k=j}^{i-2} w_{i k}^{\prime} S(k, j) \\
& =\frac{1}{b} S(i, j)-\frac{(a q+b p)}{b^{2}} S(i-1, j)+\sum_{k=j}^{i-2}\left[\frac{(-1)^{i-k} a^{i-k-2} q^{i-k-1}}{b^{i-k+1}}\left(a^{2} q+a b p-b^{2}\right) S(k, j)\right],
\end{aligned}
$$

which is of the form (24). 
For example, for $n=4$, we have

$$
\begin{aligned}
\mathscr{W}_{4} \mathscr{M}_{4}= & {\left[\begin{array}{cccc}
b & 0 & 0 & 0 \\
b p+a q & b & 0 & 0 \\
a p q+b\left(p^{2}+q\right) & b p+a q & b & 0 \\
a q\left(p^{2}+q\right)+b\left(p^{3}+2 q p\right) & a p q+b\left(p^{2}+q\right) & b p+a q & b
\end{array}\right] } \\
& \times\left[\begin{array}{ccc}
\frac{1}{b} \\
\frac{-p b+b-a q}{b^{2}} \\
\frac{-(p+q-1) b^{2}+a(p-1) q b+a^{2} q^{2}}{b^{3}}
\end{array}\right. \\
& {\left[\begin{array}{llll}
1 & 0 & 0 & 0 \\
1 & 1 & 0 & 0 \\
1 & 3 & 1 & 0 \\
1 & 7 & 6 & 1
\end{array}\right]=\mathscr{S}_{4}^{(2)} . }
\end{aligned}
$$

Since $S(n, t)=\sum_{k=1}^{n} w_{n k} \mu_{k t}, \mu_{11}=\frac{1}{b}$ and

$$
\mu_{i j}=\frac{1}{j !} \sum_{l=0}^{j}(-1)^{l}\left(\begin{array}{l}
j \\
l
\end{array}\right)\left(\frac{(j-l)^{i}}{b}-\frac{(j-l)^{i-1}(a q+b p)}{b^{2}}+\sum_{k=j}^{i-2} \frac{(-1)^{i-k} a^{i-k-2} q^{i-k-1}(j-l)^{k}}{b^{i-k+1}}\left(a^{2} q+a b p-b^{2}\right)\right)
$$

we have the following corollary.

Corollary 4.1.1. For $1<j \leqslant i$, we have

$$
\begin{aligned}
S(i, j)=\sum_{t=j}^{i} W_{i-t+1} & {\left[\frac { 1 } { j ! } \sum _ { l = 0 } ^ { j } ( - 1 ) ^ { l } ( \begin{array} { l } 
{ j } \\
{ l }
\end{array} ) \left(\frac{(j-l)^{t}}{b}-\frac{(j-l)^{t-1}(a q+b p)}{b^{2}}\right.\right.} \\
& \left.\left.+\sum_{k=j}^{t-2} \frac{(-1)^{t-k} a^{t-k-2} q^{t-k-1}(j-l)^{k}}{b^{t-k+1}}\left(a^{2} q+a b p-b^{2}\right)\right)\right] .
\end{aligned}
$$

In particular, if $j=1$ then $S(i, 1)=1$.

Definition 4. For any nonzero positive real numbers $a, b, c, d$ and any natural number $n$, an $n \times n$ matrix $\mathscr{G}_{n}=\left[\gamma_{i j}\right]$ is defined by

$$
\gamma_{i j}=\frac{1}{b} s(i, j)-\frac{(a q+b p)}{b^{2}} s(i, j+1)+\sum_{k=j+2}^{i} \frac{(-1)^{k-j} a^{k-j-2} q^{k-j-1}\left(a^{2} q+a b p-b^{2}\right)}{b^{k-j+1}} s(i, k) .
$$

Theorem 4.2. Let $\mathscr{G}_{n}$ is the matrix as in (28). For the Stirling matrix of the first kind $\mathscr{S}_{n}^{(1)}$ and second order recurrence matrix $\mathscr{W}_{n}$, we have

$$
\mathscr{S}_{n}^{(1)}=\mathscr{G}_{n} \mathscr{W}_{n}
$$


Proof. In order to prove the theorem, it is sufficient to verify $\mathscr{S}_{n}^{(1)} \mathscr{W}_{n}^{-1}=\mathscr{G}_{n}$. By virtue of the Definition 3, we see that $\gamma_{i j}=0$ for $i<j$. So, we will consider the cases $i \geqslant j$.

The cases $i=j$ and $i=j+1$ can be verified as:

$$
\gamma_{j j}=s_{j j} w_{j j}^{\prime}=\frac{1}{b}
$$

and

$$
\begin{aligned}
\gamma_{j+1, j} & =s_{j+1, j} w_{j j}^{\prime}+s_{j+1, j+1} w_{j+1, j}^{\prime} \\
& =-\frac{b(p+S(j+1, j))+a q}{b^{2}}
\end{aligned}
$$

For the last case, $i \geqslant j+2$, we get

$$
\begin{aligned}
\gamma_{i j} & =s(i, j) w_{i i}^{\prime}+s(i, j+1) w_{i, i-1}^{\prime}+\sum_{k=j+2}^{i} s(i, k) w_{k j}^{\prime} \\
& =\frac{1}{b} s(i, j)-\frac{(a q+b p)}{b^{2}} s(i, j+1)+\sum_{k=j+2}^{i} \frac{(-1)^{k-j} a^{k-j-2} q^{k-j-1}}{b^{k-j+1}}\left(a^{2} q+a b p-b^{2}\right) s(i, k)
\end{aligned}
$$

which is of the form (28).

For example, if we take $n=4$, we get

$$
\begin{aligned}
\mathscr{G}_{4} \mathscr{W}_{4}= & {\left[\begin{array}{cccc}
\frac{1}{b} & 0 & 0 & 0 \\
-\frac{p b+b+a q}{b^{2}} & \frac{1}{b} & 0 & 0 \\
\frac{(3 p-q+2) b^{2}+a(p+3) q b+a^{2} q^{2}}{b^{3}} & -\frac{b(p+3)+a q}{b^{2}} & \frac{1}{b} & 0 \\
\frac{(-11 p+6 q-6) b^{3}+a q(-6 p+q-11) b^{2}-a^{2}(p+6) q^{2} b-a^{3} q^{3}}{b^{4}} & \frac{(6 p-q+11) b^{2}+a(p+6) q b+a^{2} q^{2}}{b^{3}} & -\frac{b(p+6)+a q}{b^{2}} & \frac{1}{b}
\end{array}\right] } \\
& \times\left[\begin{array}{cccc}
b & 0 & 0 & 0 \\
b p+a q & b & 0 & 0 \\
a p q+b\left(p^{2}+q\right) & b p+a q & b & 0 \\
a q\left(p^{2}+q\right)+b\left(p^{3}+2 q p\right) & a p q+b\left(p^{2}+q\right) & b p+a q & b
\end{array}\right]
\end{aligned}
$$$$
=\left[\begin{array}{cccc}
1 & 0 & 0 & 0 \\
-1 & 1 & 0 & 0 \\
2 & -3 & 1 & 0 \\
-6 & 11 & -6 & 1
\end{array}\right]=\mathscr{S}_{4}^{(1)} .
$$

From Theorem 4.2, we can arrive at the following interesting combinatorial identity:

$$
\begin{aligned}
(n-1) !=\sum_{j=1}^{n}(-1)^{n+1}\left(\frac{1}{b} s(n, j)\right. & -\frac{(a q+b p)}{b^{2}} s(n, j+1) \\
& \left.+\sum_{k=j+2}^{n} \frac{(-1)^{k-j} a^{k-j-2} q^{k-j-1}}{b^{k-j+1}}\left(a^{2} q+a b p-b^{2}\right) s(n, k)\right) W_{j} .
\end{aligned}
$$




\section{References}

[1] Aceto, L., \& Trigiante, D. (2001). The matrices of Pascal and other greats. The American Mathematical Monthly, 108(3), 232-245.

[2] Bayat, M., \& Teimoori, H. (2000). Pascal $k$-eliminated functional matrix and its property. Linear Algebra and Its Applications, 308(1-3), 65-75.

[3] Brawer, R., \& Pirovino, M. (1992). The linear algebra of the Pascal matrix. Linear Algebra and Its Applications, 174, 13-23.

[4] Brualdi, R. A. (1977). Introductory Combinatorics. Pearson Education India.

[5] Cheon, G. S., \& Kim, J. S. (2001). Stirling matrix via Pascal matrix. Linear Algebra and its Applications, 329(1-3), 49-59.

[6] Comtet, L. (2012). Advanced Combinatorics: The Art of Finite and Infinite Expansions. Springer Science \& Business Media.

[7] Irmak, N., \& Köme, C. (2021). Linear Algebra of the Lucas Matrix. Hacettepe Journal of Mathematics and Statistics, 50(2), 549-558.

[8] Lee, G. Y., \& Cho, S. H. (2008). The generalized Pascal matrix via the generalized Fibonacci matrix and the generalized Pell matrix. Journal of the Korean Mathematical Society, 45(2), 479-491.

[9] Lee, G. Y., Kim, J. S., \& Cho, S. H. (2003). Some combinatorial identities via Fibonacci numbers. Discrete Applied Mathematics, 130(3), 527-534.

[10] Quintana, Y., Ramírez, W., \& Urieles G., A. (2019). Generalized Apostol-type polynomial matrix and its algebraic properties. Mathematical Reports, 21(71), 2, 249-264.

[11] Stanica, P. (2005). Cholesky factorizations of matrices associated with $r$-order recurrent sequences. Integers: Electronic Journal of Combinatorial Number Theory, 5(2), A16.

[12] Stanimirović, P., Nikolov, J., \& Stanimirović, I. (2008). A generalization of Fibonacci and Lucas matrices. Discrete Applied Mathematics, 156(14), 2606-2619.

[13] Zhang, Z. (1997). The linear algebra of the generalized Pascal matrix. Linear Algebra and Its Applications, 250, 51-60.

[14] Zhang, Z., \& Wang, X. (2007). A factorization of the symmetric Pascal matrix involving the Fibonacci matrix. Discrete Applied Mathematics, 155(17), 2371-2376.

[15] Zhang, Z., \& Zhang, Y. (2007). The Lucas matrix and some combinatorial identities. Indian Journal of Pure and Applied Mathematics, 38(5), 457-465. 